\title{
Higher-Order Numerical Solution of Two-Dimensional Coupled Burgers' Equations
}

\author{
T. Zhanlav' ${ }^{1}$ O. Chuluunbaatar ${ }^{1,2}$, V. Ulziibayar ${ }^{1,3}$ \\ ${ }^{1}$ Institute of Mathematics, National University of Mongolia, Ulaanbaatar, Mongolia \\ 2Joint Institute for Nuclear Research, Moscow, Russia \\ ${ }^{3}$ School of Applied Sciences, Mongolian University of Science and Technology, Ulaanbaatar, Mongolia \\ Email: tzhanlav@yahoo.com,chuka@jinr.ru,v_ulzii@yahoo.com
}

Received 10 October 2015; accepted 13 June 2016; published 16 June 2016

Copyright (C) 2016 by authors and Scientific Research Publishing Inc.

This work is licensed under the Creative Commons Attribution International License (CC BY). http://creativecommons.org/licenses/by/4.0/

(c) (i) Open Access

\begin{abstract}
We proposed a higher-order accurate explicit finite-difference scheme for solving the two-dimensional heat equation. It has a fourth-order approximation in the space variables, and a secondorder approximation in the time variable. As an application, we developed the proposed numerical scheme for solving a numerical solution of the two-dimensional coupled Burgers' equations. The main advantages of our scheme are higher accurate accuracy and facility to implement. The good accuracy of the proposed numerical scheme is tested by comparing the approximate numerical and the exact solutions for several two-dimensional coupled Burgers' equations.
\end{abstract}

\section{Keywords}

Two-Dimensional Coupled Burgers' Equation, Hopf-Cole Transformation, Higher-Order Accurate Numerical Schemes

\section{Introduction}

The Burgers' equation is an important non-linear parabolic partial differential equation widely used to model several physical flow phenomena in fluid dynamics teaching and in engineering such as turbulence, boundary layer behaviour, shock wave formation and mass transport [1]. Due to its wide range of applicability, several researchers, both scientists and engineers, have been interested in studying the properties of the two-dimensional coupled Burgers' equation (TDCBE) using various numerical techniques.

There exist many different explicit and implicit numerical schemes with second-order approximation in the 
space variables, and a first or second-order approximation in the time variable. For example in [2]-[5], the Crank-Nicolson scheme using the different fully/semi implicit finite-difference methods for the numerical solution of the TDCBE was applied. The implicit logarithmic and local discontinuous Galerkin finite-difference methods for the numerical solution of the TDCBE are proposed in [6] [7]. Also in [4] an explicit scheme using the finite-difference method was applied.

The implicit finite-difference methods with forth-order approximation in the space variables, and a secondorder approximation in the time variable are proposed in [8] [9]. These methods based on the Crank-Nicolson scheme with Padé approximation of the finite-difference operator, and hybrid Crank-Nicolson Du Fort and Frankel scheme, respectively. However, the implicit methods on each time layer required to solve an algebraic system. In multidimensional case of the TDHE, it requires large calculation time for solving the algebraic systems till final time layer $t=T$, even taking into account the band structure of the matrices [10].

The aim of the present paper is to construct a new stable and explicit finite-difference scheme to solve the two-dimensional heat equation (TDHE) with Robin boundary conditions. The proposed scheme has a fourthorder approximation in the space variables, and a second-order approximation in the time variable. We developed the proposed scheme for solving a numerical solution of the TDCBE, which comes into the TDHE by the application of the Hopf-Cole transformation.

It is known that the time step of the explicit time-marching schemes must satisfy the so-called CourantFriedrichs-Lewy condition, which usually enforces a limiting constraint on the time step. However, the main advantages of our explicit scheme considered are saving computing time and memory, and making parallelization easier compared to the other numerical methods applied to the TDHE.

The accuracy of the proposed numerical scheme is examined by comparing the numerical and exact solutions of the several TDCBE. The numerical results are found in good agreement with exact solutions for a wide rang of the Reynolds number and confirm the approximation orders of the proposed scheme. We also compared the efficiency of the proposed scheme and implicit fourth-order finite-difference method [8]. Both methods are comparable by the convergence of the solutions and total calculation times.

The structure of the paper is as follows. In Section 2, we present reductions of the TDCBE to a TDHE. The explicit fourth-order accurate finite-difference scheme for solving the TDHE and the fourth-order accurate finite-difference schemes for solving the TDCBE are given in Section 3. Numerical results are discussed in Section 4.

\section{The Statement of the Problem}

The TDCBE is given by

$$
\begin{aligned}
& \frac{\partial u(x, y, t)}{\partial t}+u(x, y, t) \frac{\partial u(x, y, t)}{\partial x}+v(x, y, t) \frac{\partial u(x, y, t)}{\partial y}=v\left(\frac{\partial^{2} u(x, y, t)}{\partial x^{2}}+\frac{\partial^{2} u(x, y, t)}{\partial y^{2}}\right) \\
& \frac{\partial v(x, y, t)}{\partial t}+u(x, y, t) \frac{\partial v(x, y, t)}{\partial x}+v(x, y, t) \frac{\partial v(x, y, t)}{\partial y}=v\left(\frac{\partial^{2} v(x, y, t)}{\partial x^{2}}+\frac{\partial^{2} v(x, y, t)}{\partial y^{2}}\right)
\end{aligned}
$$

subject to the initial conditions

$$
\begin{aligned}
& u(x, y, 0)=\varphi_{1}(x, y), \quad(x, y) \in \Omega, \\
& v(x, y, 0)=\varphi_{2}(x, y), \quad(x, y) \in \Omega,
\end{aligned}
$$

and Dirichlet boundary conditions

$$
\begin{array}{ll}
u(x, y, t)=\zeta(x, y, t), \quad(x, y) \in \partial \Omega, & 0<t \leq T, \\
v(x, y, t)=\xi(x, y, t), \quad(x, y) \in \partial \Omega, & 0<t \leq T,
\end{array}
$$

and the potential symmetry condition

$$
\frac{\partial u(x, y, t)}{\partial y}=\frac{\partial v(x, y, t)}{\partial x}, \quad(x, y) \in \Omega, \quad 0 \leq t \leq T .
$$


Here $\Omega=\{(x, y): a \leq x \leq b, c \leq y \leq d\}$ is the computational domain, and $\partial \Omega$ is its boundary; $u(x, y, t)$ and $v(x, y, t)$ are the velocity components to be determined; $\varphi_{1}(x, y), \varphi_{2}(x, y), \zeta(x, y, t)$ and $\xi(x, y, t)$ are known functions; $v^{-1}$ is the Reynolds number.

Using the Hopf-Cole transformations [7] [8]

$$
\begin{aligned}
& u(x, y, t)=-2 v \frac{1}{\theta(x, y, t)} \frac{\partial \theta(x, y, t)}{\partial x}, \\
& v(x, y, t)=-2 v \frac{1}{\theta(x, y, t)} \frac{\partial \theta(x, y, t)}{\partial y},
\end{aligned}
$$

Equations (1) (2) are reduced to the TDHE

$$
\frac{\partial \theta(x, y, t)}{\partial t}-v\left(\frac{\partial^{2} \theta(x, y, t)}{\partial x^{2}}+\frac{\partial^{2} \theta(x, y, t)}{\partial y^{2}}\right)-C(t) \theta(x, y, t)=0,
$$

where $C(t)$ is an arbitrary function depending on $t$ only.

Theorem 1 [7]. Let $\theta(x, y, t)$ be the solution of Equation (10), the functions $u(x, y, t)$ and $v(x, y, t)$ are defined in Equations (8) and (9). Then $u(x, y, t)$ and $v(x, y, t)$ are independent of the function $C(t)$.

By the above theorem, we can choose $C(t)=0$, and Equation (10) is simplified to

$$
\frac{\partial \theta(x, y, t)}{\partial t}=v\left(\frac{\partial^{2} \theta(x, y, t)}{\partial x^{2}}+\frac{\partial^{2} \theta(x, y, t)}{\partial y^{2}}\right) .
$$

The initial conditions (3), (4) and boundary conditions (5), (6) lead to

$$
\begin{aligned}
& \theta(x, y, 0)=\Phi(x, y), \\
& \left.\frac{\partial \theta(x, y, t)}{\partial x}\right|_{x=a}+\frac{\zeta(a, y, t)}{2 v} \theta(a, y, t)=0, \\
& \left.\frac{\partial \theta(x, y, t)}{\partial x}\right|_{x=b}+\frac{\zeta(b, y, t)}{2 v} \theta(b, y, t)=0, \\
& \left.\frac{\partial \theta(x, y, t)}{\partial y}\right|_{y=c}+\frac{\xi(x, c, t)}{2 v} \theta(x, c, t)=0, \\
& \left.\frac{\partial \theta(x, y, t)}{\partial y}\right|_{y=d}+\frac{\xi(x, d, t)}{2 v} \theta(x, d, t)=0,
\end{aligned}
$$

respectively. Here the initial-condition function $\Phi(x, y)$ has the form [7] [8]

$$
\Phi(x, y)=\exp \left(-\frac{1}{2 v} \int_{a}^{x} \varphi_{1}(s, y) \mathrm{d} s-\frac{1}{2 v} \int_{c}^{y} \varphi_{2}(a, s) \mathrm{d} s\right) .
$$

Thus, the TDCBE (1)-(6) are fully reduced to TDHE (11) with the initial and boundary conditions (12)-(16).

\section{The Fourth-Order Accurate Explicit Finite-Difference Scheme}

For the TDHE (11)-(16), we consider the following eleven-points explicit finite-difference scheme:

$$
\begin{aligned}
F(\Theta) \equiv & \frac{A \Theta_{i, j}^{n+1}-(A+B) \Theta_{i, j}^{n}+B \Theta_{i, j}^{n-1}}{\tau}-C \frac{\Theta_{i-1, j}^{n}-2 \Theta_{i, j}^{n}-\Theta_{i+1, j}^{n}}{h_{x}^{2}}-C \frac{\Theta_{i, j-1}^{n}-2 \Theta_{i, j}^{n}+\Theta_{i, j+1}^{n}}{h_{y}^{2}} \\
& -D\left(\frac{1}{h_{x}^{2}}+\frac{1}{h_{y}^{2}}\right)\left(\Theta_{i-1, j-1}^{n}+\Theta_{i-1, j+1}^{n}+\Theta_{i+1, j-1}^{n}+\Theta_{i+1, j+1}^{n}-4 \Theta_{i, j}^{n}\right) \\
& i=1, \cdots, N-1, \quad j=1, \cdots, M-1, \quad N h_{x}=b-a, \quad M h_{y}=d-c, \quad n=1,2, \cdots
\end{aligned}
$$


Here and throughout the work, $\Theta_{i, j}^{n}$ is the approximate solution of the $\theta_{i, j}^{n}=\theta\left(x_{i}, y_{j}, t_{n}\right)$ at the mesh point ( $x_{i}=i h, y_{j}=j h, t_{n}=n \tau$ ), where $h_{x}$ and $h_{y}$ are spatial steps by $x$ and $y, \tau$ is a time step, $A, B, C$ and $D$ are unknown coefficients. Let $z_{i, j}^{n}=\Theta_{i, j}^{n}-\theta_{i, j}^{n}$ be the error function. In this term the scheme (18) has the form

$$
F(z)=\psi_{i, j}^{n},
$$

where $\psi_{i, j}^{n}$ is an approximation error and

$$
\psi_{i, j}^{n}=F(\theta) .
$$

We suppose that the solution of Equations (11)-(16) is a sufficiently smooth function with respect to $x, y$ and $t$. Using the Taylor expansions of $\theta_{i, j}^{n \pm 1}, \theta_{i \pm 1, j}^{n}, \theta_{i, j \pm 1}^{n}$ and $\theta_{i \pm 1, j \pm 1}^{n}$ at the point $\left(x_{i}, y_{j}, t_{n}\right)$, and an identity

$$
\frac{\partial^{m} \theta(x, y, t)}{\partial t^{m}}=v^{m}\left(\frac{\partial^{2}}{\partial x^{2}}+\frac{\partial^{2}}{\partial x^{2}}\right)^{m} \theta(x, y, t), \quad m \geq 0,
$$

we have

$$
\begin{aligned}
& \psi_{i, j}^{n}=\left(\left[(A-B) v-C-2 D\left(1+\frac{h_{x}^{2}}{h_{y}^{2}}\right)\right] \frac{\partial^{2} \theta(x, y, t)}{\partial x^{2}}+\left[(A-B) v-C-2 D\left(1+\frac{h_{y}^{2}}{h_{x}^{2}}\right)\right] \frac{\partial^{2} \theta(x, y, t)}{\partial y^{2}}\right. \\
& +\left[(A+B) \frac{\tau v^{2}}{2}-C \frac{h_{x}^{2}}{12}-D\left(\frac{h_{x}^{2}}{6}+\frac{h_{x}^{4}}{6 h_{y}^{2}}\right)\right] \frac{\partial^{4} \theta(x, y, t)}{\partial x^{4}}+\left[(A+B) \frac{\tau v^{2}}{2}-C \frac{h_{y}^{2}}{12}-D\left(\frac{h_{y}^{2}}{6}+\frac{h_{y}^{4}}{6 h_{x}^{2}}\right)\right] \frac{\partial^{4} \theta(x, y, t)}{\partial y^{4}} \\
& \left.+\left[(A+B) \tau v^{2}-D\left(h_{x}^{2}+h_{y}^{2}\right)\right] \frac{\partial^{4} \theta(x, y, t)}{\partial x^{2} \partial y^{2}}\right)\left.\right|_{x=x_{i}, y=y_{j}, t=t_{n}}+O\left(h_{x}^{4}+h_{y}^{4}+h_{x}^{2} h_{y}^{2}+\frac{h_{x}^{6}}{h_{y}^{2}}+\frac{h_{y}^{6}}{h_{x}^{2}}\right) .
\end{aligned}
$$

Equating the coefficients of the partial derivatives to zero in (22), we obtain following system of equations

$$
\left\{\begin{array}{l}
(A-B) v=C+2 D\left(1+\frac{h_{x}^{2}}{h_{y}^{2}}\right)=C+2 D\left(1+\frac{h_{y}^{2}}{h_{x}^{2}}\right), \\
(A+B) \tau v^{2}=C \frac{h_{x}^{2}}{6}+D\left(\frac{h_{x}^{2}}{3}+\frac{h_{x}^{4}}{3 h_{y}^{2}}\right)=C \frac{h_{y}^{2}}{6}+D\left(\frac{h_{y}^{2}}{3}+\frac{h_{y}^{4}}{3 h_{x}^{2}}\right), \\
(A+B) \tau v^{2}=D\left(h_{x}^{2}+h_{y}^{2}\right) .
\end{array}\right.
$$

The above system has a unique solution if $h=h_{x}=h_{y}$ :

$$
C=8 D=\frac{4 A \alpha v}{1+3 \alpha}, \quad B=A \frac{1-3 \alpha}{1+3 \alpha},
$$

where $\alpha=\frac{2 \tau v}{h^{2}}$. Using (24) and the higher-order Taylor expansions of the $\theta(x, y, t)$ at the point $\left(x_{i}, y_{j}, t_{n}\right)$ we obtain

$$
\begin{aligned}
\psi_{i, j}^{n}= & D \frac{h^{4}}{2}\left(\left(\alpha^{2}-\frac{1}{15}\right)\left(\frac{\partial^{6} \theta(x, y, t)}{\partial x^{6}}+\frac{\partial^{6} \theta(x, y, t)}{\partial y^{6}}\right)\right. \\
& \left.+3\left(\alpha^{2}-\frac{1}{9}\right)\left(\frac{\partial^{6} \theta(x, y, t)}{\partial x^{4} \partial y^{2}}+\frac{\partial^{6} \theta(x, y, t)}{\partial x^{2} \partial y^{4}}\right)\right)\left.\right|_{x=x_{i}, y=y_{j}, t=t_{n}}+O\left(\tau^{3}+h^{6}\right) .
\end{aligned}
$$

One can see that, the condition [11]

$$
\alpha=\frac{2 \tau \nu}{h^{2}}=\sqrt{\frac{1}{15}}
$$

does not improve the order of the scheme (18), i.e., the truncation error of the scheme (18) is of the order of 
$\psi_{i, j}^{n}=O\left(\tau^{2}+h^{4}\right)$ for any $\alpha$. If $A=1$, the scheme (18) is simplified to the canonical form:

$$
\begin{aligned}
\Theta_{i, j}^{n+1}= & -\frac{1-3 \alpha}{1+3 \alpha} \Theta_{i, j}^{n-1}+\frac{2-10 \alpha^{2}}{1+3 \alpha} \Theta_{i, j}^{n} \\
& +\frac{2 \alpha^{2}}{1+3 \alpha}\left(\Theta_{i-1, j}^{n}+\Theta_{i+1, j}^{n}+\Theta_{i, j-1}^{n}+\Theta_{i, j+1}^{n}+\frac{1}{4}\left(\Theta_{i-1, j-1}^{n}+\Theta_{i-1, j+1}^{n}+\Theta_{i+1, j-1}^{n}+\Theta_{i+1, j+1}^{n}\right)\right),
\end{aligned}
$$

To find the stability condition of the scheme (27), we seek the partial solution in the form:

$$
\Theta_{i, j}^{n}=q^{n} \exp (\imath i h \psi) \exp (\imath j h \phi)
$$

From (27) we have

$$
\begin{aligned}
& (1+3 \alpha) q^{2}-2 b q+1-3 \alpha=0, \\
& b=1-(9-A) \alpha^{2}, \\
& 1 \leq A=(\cos (h \psi)+2)(\cos (h \phi)+2) \leq 9 .
\end{aligned}
$$

We have following theorem:

Theorem 2 [10] Let $a>0, b$ and $c$ are real numbers. Then roots of a quadratic equation $a q^{2}+2 b q+c=0$ satisfy the condition $\left|q_{1,2}\right| \leq 1$ if and only if

$$
\frac{c}{a} \leq 1, \quad 2|b| \leq a+c .
$$

Using the conditions (30), we obtain

$$
\left\{\begin{array} { l } 
{ \frac { c } { a } = \frac { 1 - 3 \alpha } { 1 + 3 \alpha } \leq 1 , } \\
{ a + c = 1 + 3 \alpha + 1 - 3 \alpha \geq 2 b = 2 ( 1 - ( 9 - A ) \alpha ^ { 2 } ) , } \\
{ a + c = 1 + 3 \alpha + 1 - 3 \alpha \geq - 2 b = 2 ( ( 9 - A ) \alpha ^ { 2 } - 1 ) , }
\end{array} \Rightarrow \left\{\begin{array}{l}
0 \leq \alpha, \\
A \leq 9, \\
\alpha \leq \sqrt{\frac{2}{9-A}}
\end{array}\right.\right.
$$

The last inequality is true for any $A$ under condition $\alpha \leq \frac{1}{2}$ or

$$
\tau \leq \frac{h^{2}}{4 v}
$$

The scheme (27) at $\alpha=\frac{1}{3}$ (or $B=0$ ) is a two-layer scheme in time, while at $\alpha \neq \frac{1}{3}$ (or $B \neq 0$ ) is a threelayer one. Hence, if $\alpha \neq \frac{1}{3}$ in order to find $\Theta_{i, j}^{2}$ at level two, two values $\Theta_{i, j}^{0}$ and $\Theta_{i, j}^{1}$ are required. Using the Taylor expansion of $\theta(x, y, t)$ at point $(x, y, 0)$ and Equation (11) we obtain

$$
\theta(x, y, t)=\theta(x, y, 0)+\tau v\left(\frac{\partial^{2} \theta(x, y, 0)}{\partial x^{2}}+\frac{\partial^{2} \theta(x, y, 0)}{\partial y^{2}}\right)+O\left(\tau^{2}\right) .
$$

From the initial condition (12) and Taylor expansion (33), we find $\Theta_{i, j}^{1}$ with the accuracy $O\left(\tau^{3}\right)$

$$
\Theta_{i, j}^{1}=\Phi\left(x_{i}, y_{j}\right)+\left.\tau \nu\left(\frac{\partial^{2} \Phi(x, y)}{\partial x^{2}}+\frac{\partial^{2} \Phi(x, y)}{\partial y^{2}}\right)\right|_{x=x_{i}, y=y_{j}} \quad, i=0, \cdots, N, \quad j=0, \cdots, M .
$$

From the Robin boundary conditions (13)-(16) using the asymmetric fourth-order finite-difference approximations of the first spatial derivative [12], we find $\Theta_{0, j}^{n+1}, \Theta_{N, j}^{n+1}, \Theta_{i, 0}^{n+1}$ and $\Theta_{i, M}^{n+1}$ 


$$
\begin{aligned}
& \Theta_{0, j}^{n+1}=\frac{48 \Theta_{1, j}^{n+1}-36 \Theta_{2, j}^{n+1}+16 \Theta_{3, j}^{n+1}-3 \Theta_{4, j}^{n+1}}{25-\frac{12 h}{2 v} \zeta\left(a, y_{j}, t_{n+1}\right)}, \quad j=1, \cdots, M-1, \\
& \Theta_{N, j}^{n+1}=-\frac{3 \Theta_{N-4, j}^{n+1}-16 \Theta_{N-3, j}^{n+1}+36 \Theta_{N-2, j}^{n+1}-48 \Theta_{N-1, j}^{n+1}}{25+\frac{12 h}{2 v} \zeta\left(b, y_{j}, t_{n+1}\right)}, \\
& \Theta_{i, 0}^{n+1}=\frac{48 \Theta_{i, 1}^{n+1}-36 \Theta_{i, 2}^{n+1}+16 \Theta_{i, 3}^{n+1}-3 \Theta_{i, 4}^{n+1}}{25-\frac{12 h}{2 v} \xi\left(x_{i}, c, t_{n+1}\right)}, \quad i=1, \cdots, N-1 \\
& \Theta_{i, M}^{n+1}=-\frac{3 \Theta_{i, M-4}^{n+1}-16 \Theta_{i, M-3}^{n+1}+36 \Theta_{i, M-2}^{n+1}-48 \Theta_{i, M-1}^{n+1}}{25+\frac{12 h}{2 v} \xi\left(x_{i}, d, t_{n+1}\right)} .
\end{aligned}
$$

Now we need to calculate values of the vertex points $\Theta_{0,0}^{n+1}, \Theta_{N, 0}^{n+1}, \Theta_{0, M}^{n+1}$ and $\Theta_{N, M}^{n+1}$. Each value of these points can be calculated using the boundary conditions (13)-(16) and a similar formula to (35) by direction $x$ or $y$ or a middle value of the values by the both directions. Below we presented formulas which used only the boundary conditions (13), (14):

$$
\begin{aligned}
\Theta_{0,0}^{n+1} & =\frac{48 \Theta_{1,0}^{n+1}-36 \Theta_{2,0}^{n+1}+16 \Theta_{3,0}^{n+1}-3 \Theta_{4,0}^{n+1}}{25-\frac{12 h}{2 v} \zeta\left(a, c, t_{n+1}\right)}, \\
\Theta_{N, 0}^{n+1} & =-\frac{3 \Theta_{N-4,0}^{n+1}-16 \Theta_{N-3,0}^{n+1}+36 \Theta_{N-2,0}^{n+1}-48 \Theta_{N-1,0}^{n+1}}{25+\frac{12 h}{2 v} \zeta\left(b, c, t_{n+1}\right)}, \\
\Theta_{0, M}^{n+1} & =\frac{48 \Theta_{1, M}^{n+1}-36 \Theta_{2, M}^{n+1}+16 \Theta_{3, M}^{n+1}-3 \Theta_{4, M}^{n+1}}{25-\frac{12 h}{2 v} \zeta\left(a, d, t_{n+1}\right)}, \\
\Theta_{N, M}^{n+1} & =-\frac{3 \Theta_{N-4, M}^{n+1}-16 \Theta_{N-3, M}^{n+1}+36 \Theta_{N-2, M}^{n+1}-48 \Theta_{N-1, M}^{n+1}}{25+\frac{12 h}{2 v} \zeta\left(b, d, t_{n+1}\right)} .
\end{aligned}
$$

Thus, we find $\Theta_{i, j}^{n}$ for $i=0, \cdots, N$ and $j=0, \cdots, M$ by Formulas (27), (35) and (36).

The higher-order finite-difference schemes presented in our previous papers [13]-[15] are applied for finding solutions $u(x, y, t), v(x, y, t)$ of Equations (1)-(6). We used the following fourth-order finite-difference scheme [13]:

$$
\Theta_{i-1, j}^{n} U_{i-1, j}^{n}+4 \Theta_{i, j}^{n} U_{i, j}^{n}+\Theta_{i+1, j}^{n} U_{i+1, j}^{n}=-\frac{6 v}{h}\left(\Theta_{i+1, j}^{n}-\Theta_{i-1, j}^{n}\right), i=1, \cdots, N-1, j=0, \cdots, M,
$$

with boundary conditions

$$
U_{0, j}^{n}=\zeta\left(a, y_{j}, t_{n}\right), \quad U_{N, j}^{n}=\zeta\left(b, y_{j}, t_{n}\right), \quad j=0, \cdots, M .
$$

Here $U_{i, j}^{n}$ is an approximate solution of $u\left(x_{i}, y_{j}, t_{n}\right)$. In a similar way we obtain

$$
\Theta_{i, j-1}^{n} V_{i, j-1}^{n}+4 \Theta_{i, j}^{n} V_{i, j}^{n}+\Theta_{i, j+1}^{n} V_{i, j+1}^{n}=-\frac{6 v}{h}\left(\Theta_{i, j+1}^{n}-\Theta_{i, j-1}^{n}\right), j=1, \cdots, M-1, i=0, \cdots, N,
$$

with boundary conditions

$$
V_{i, 0}^{n}=\xi\left(x_{i}, c, t_{n}\right), \quad V_{i, M}^{n}=\xi\left(x_{i}, d, t_{n}\right), \quad i=0, \cdots, N .
$$

Here $V_{i, j}^{n}$ is an approximate solution of $v\left(x_{i}, y_{j}, t_{n}\right)$. The three-diagonal systems (37), (38) and (39), (40) are solved by the efficient elimination method [16]. 


\section{Numerical Results}

Two exact solvable TDCBEs (1)-(6) are solved to show demonstrate the efficiency and robustness of the proposed schemes. To analyze the convergence of the proposed schemes, we used the maximum absolute errors of the solutions $\theta(x, y, t), u(x, y, t)$ and $v(x, y, t)$ :

$$
\begin{aligned}
\left\|e_{\theta}\right\|_{\infty, h} & =\max _{0 \leq i \leq N, 0 \leq j \leq M}\left|\theta\left(x_{i}, y_{j}, t\right)-\Theta\left(x_{i}, y_{j}, t\right)\right|, \\
\left\|e_{u}\right\|_{\infty, h} & =\max _{0 \leq i \leq N, 0 \leq j \leq M}\left|u\left(x_{i}, y_{j}, t\right)-U\left(x_{i}, y_{j}, t\right)\right|, \\
\left\|e_{v}\right\|_{\infty, h} & =\max _{0 \leq i \leq N, 0 \leq j \leq M}\left|v\left(x_{i}, y_{j}, t\right)-V\left(x_{i}, y_{j}, t\right)\right| .
\end{aligned}
$$

The order (or Runge coefficient) of convergence of the proposed schemes is defined by the double-crowding spatial grids

$$
\operatorname{Order}_{\theta, u, v}=\log _{2}\left(\frac{\left\|e_{\theta, u, v}\right\|_{\infty, h}}{\left\|e_{\theta, u, v}\right\|_{\infty, h / 2}}\right)
$$

The initial and boundary conditions (3), (4), (12) and (5), (6), (13)-(16) for the solutions $u(x, y, t), v(x, y, t)$, $\theta(x, y, t)$ are taken from the analytical solutions. The computational domain is $\Omega=\{(x, y): 0 \leq x \leq 1,0 \leq y \leq 1\}$.

All calculations were performed in double-precision arithmetic on a AMD Phenom II X6 processor using Intel FORTRAN Compiler.

Example 1. ([7] [8]). In this example, we solve the two-dimensional Burgers Equations (1), (2), for which the exact solutions are

$$
\begin{aligned}
& u(x, y, t)=-2 v \frac{2 \pi \exp \left(-5 v \pi^{2} t\right) \cos (2 \pi x) \sin (\pi y)}{2+\exp \left(-5 v \pi^{2} t\right) \sin (2 \pi x) \sin (\pi y)}, \\
& v(x, y, t)=-2 v \frac{\pi \exp \left(-5 v \pi^{2} t\right) \sin (2 \pi x) \cos (\pi y)}{2+\exp \left(-5 v \pi^{2} t\right) \sin (2 \pi x) \sin (\pi y)} .
\end{aligned}
$$

The initial and boundary conditions are taken from the exact solutions. We solve the TDHE (11), for which the exact solution is

$$
\theta(x, y, t)=\frac{2+\exp \left(-5 v \pi^{2} t\right) \sin (2 \pi x) \sin (\pi y)}{2}
$$

The initial (12) and boundary conditions (13)-(16) are taken from the exact solutions.

The convergence of the solutions $\theta(x, y, t), u(x, y, t), v(x, y, t)$ versus the inverse of the Reynolds number $v$ and the numbers of grid $N, M$ are presented in Table 1. To show that the method is fourth-order accurate in space, we fix the time step $\tau$ as 0.0001 that in each the numbers of grid $N=M \leq 80$ holds the stability condition (32). The orders of convergence of the proposed schemes are consistent with the theoretical expectations $O\left(h^{4}\right)$.

In Table 2 we compared the efficiency of the proposed scheme with the time step $\tau=\frac{h^{2}}{4 v}$ and implicit the fourth-order finite-difference method with the time step $\tau=0.01$ [8] at $v=0.01$. From this Table the both methods are comparable by the convergence of the solution $\theta(x, y, t)$ and total calculation times.

Example 2 ([7] [8] [17]). In this example, we solve the two-dimensional Burgers Equations (1), (2), for which the exact solutions are

$$
u(x, y, t)=\frac{3}{4}-\frac{1}{4}\left(1+\exp \left(\frac{-4 x+4 y-t}{32 v}\right)\right)^{-1},
$$


Table 1. The convergence of the solutions $\theta(x, y, t), u(x, y, t), v(x, y, t)$ and their corresponding orders of convergence for the Example 1 at $T=1, \tau=0.0001$ versus the parameter $v$ and the numbers of grid $N, M$. The first column shows the parameter $v$, the second ones displays the numbers of grid $N, M$. The third, fifth and seventh columns display the maximum absolute error $\left\|e_{\theta}\right\|_{\infty, h},\left\|e_{u}\right\|_{\infty, h},\left\|e_{v}\right\|_{\infty, h}$, while the second, forth, and sixth columns present their orders of convergence, respectively. The factor $x$ in the brackets denotes $10^{x}$.

\begin{tabular}{|c|c|c|c|c|c|c|c|}
\hline \multirow[b]{2}{*}{ Example 1.} & \multirow[b]{2}{*}{$N=M$} & \multicolumn{2}{|c|}{$\theta=\theta(x, y, t)$} & \multicolumn{2}{|c|}{$u=u(x, y, t)$} & \multicolumn{2}{|c|}{$v=v(x, y, t)$} \\
\hline & & $\left\|e_{\theta}\right\|_{\infty, h}$ & $\operatorname{Order}_{\theta}$ & $\left\|e_{u}\right\|_{\infty, h}$ & $\operatorname{Order}_{u}$ & $\left\|e_{v}\right\|_{\infty, h}$ & Order $_{1}$ \\
\hline \multirow[t]{4}{*}{$v=0.001$} & 10 & $0.269(-2)$ & & $0.441(-4)$ & & $0.177(-4)$ & \\
\hline & 20 & $0.209(-3)$ & 3.687 & $0.611(-5)$ & 2.853 & $0.129(-5)$ & 3.782 \\
\hline & 40 & $0.138(-4)$ & 3.917 & $0.607(-6)$ & 3.332 & $0.792(-7)$ & 4.027 \\
\hline & 80 & $0.890(-6)$ & 3.959 & $0.474(-7)$ & 3.676 & $0.511(-8)$ & 3.955 \\
\hline \multirow[t]{4}{*}{$v=0.01$} & 10 & $0.462(-2)$ & & $0.510(-3)$ & & $0.220(-3)$ & \\
\hline & 20 & $0.518(-3)$ & 3.156 & $0.716(-4)$ & 2.831 & $0.253(-4)$ & 3.120 \\
\hline & 40 & $0.365(-4)$ & 3.823 & $0.536(-5)$ & 3.739 & $0.179(-5)$ & 3.819 \\
\hline & 80 & $0.235(-5)$ & 3.955 & $0.351(-6)$ & 3.931 & $0.115(-6)$ & 3.953 \\
\hline \multirow[t]{4}{*}{$v=0.1$} & 10 & $0.164(-2)$ & & $0.800(-3)$ & & $0.102(-3)$ & \\
\hline & 20 & $0.177(-3)$ & 3.207 & $0.863(-4)$ & 3.212 & $0.115(-4)$ & 3.159 \\
\hline & 40 & $0.125(-4)$ & 3.827 & $0.607(-5)$ & 3.829 & $0.812(-6)$ & 3.825 \\
\hline & 80 & $0.820(-6)$ & 3.930 & $0.398(-6)$ & 3.929 & $0.523(-7)$ & 3.955 \\
\hline
\end{tabular}

Table 2. Comparison of the maximum absolute error $\left\|e_{\theta}\right\|_{\infty, h}$ and CPU-time of the proposed scheme with $\tau=\frac{h^{2}}{4 v}$ and implicit the fourth-order finite-difference method [8] with $\tau=0.01$. Here considered the Example 1 at $v=0.01, T=1$. The factor $x$ in the brackets denotes $10^{x}$.

\begin{tabular}{clccccc}
\hline Example 1. & \multicolumn{3}{c}{ Proposed scheme at $\tau=\frac{h^{2}}{4 v}$} & \multicolumn{2}{c}{ The scheme in [8] at $\tau=0.01$} \\
\hline$N=M$ & $\left\|e_{\theta}\right\|_{\infty, h}$ & Order $_{\theta}$ & CPU-time, sec & $\left\|e_{\theta}\right\|_{\infty, h}$ & Order $_{\theta}$ & CPU-time, sec \\
\hline 10 & $0.458(-2)$ & & 0.000 & $0.584(-3)$ & 0.000 \\
20 & $0.579(-3)$ & 2.982 & 0.000 & $0.324(-4)$ & 4.171 & 0.031 \\
40 & $0.425(-4)$ & 3.768 & 0.015 & $0.200(-5)$ & 4.017 & 0.124 \\
80 & $0.276(-5)$ & 3.944 & 0.218 & $0.249(-6)$ & 3.006 & 0.483 \\
\hline
\end{tabular}

$$
v(x, y, t)=\frac{3}{4}+\frac{1}{4}\left(1+\exp \left(\frac{-4 x+4 y-t}{32 v}\right)\right)^{-1} .
$$

The initial and boundary conditions are taken from the exact solutions. We solve the TDHE (11), for which the exact solution is

$$
\theta(x, y, t)=\frac{1}{2} \exp \left(-\frac{12 x+12 y-9 t}{32 v}\right)\left(1+\exp \left(\frac{4 x-4 y+t}{32 v}\right)\right) .
$$

The initial (12) and boundary conditions (13)-(16) are taken from the exact solutions.

The solutions (45) are so-called shock solutions of the TDCBE. It is well known that one of the difficulties in 
Table 3. The same as in Table 1, but for for the Example 2. The factor $x$ in the brackets denotes $10^{x}$.

\begin{tabular}{|c|c|c|c|c|c|}
\hline \multirow[b]{2}{*}{ Example 2.} & \multirow[b]{2}{*}{$N=M$} & \multicolumn{2}{|c|}{$u=u(x, y, t)$} & \multicolumn{2}{|c|}{$v=v(x, y, t)$} \\
\hline & & $\left\|e_{u}\right\|_{\infty, h}$ & $\operatorname{Order}_{u}$ & $\left\|e_{v}\right\|_{\infty, h}$ & $\operatorname{Order}_{v}$ \\
\hline$v=0.01$ & 80 & $0.502(-2)$ & & $0.455(-1)$ & \\
\hline \multirow[t]{3}{*}{$\tau=0.00001$} & 160 & $0.489(-3)$ & 3.360 & $0.258(-2)$ & 4.138 \\
\hline & 320 & $0.399(-4)$ & 3.616 & $0.155(-3)$ & 4.057 \\
\hline & 640 & $0.286(-5)$ & 3.801 & $0.938(-5)$ & 4.048 \\
\hline$v=0.05$ & 20 & $0.209(-2)$ & & $0.140(-1)$ & \\
\hline \multirow[t]{3}{*}{$\tau=0.00001$} & 40 & $0.158(-3)$ & 3.727 & $0.813(-3)$ & 4.107 \\
\hline & 80 & $0.111(-4)$ & 3.828 & $0.484(-4)$ & 4.070 \\
\hline & 160 & $0.740(-6)$ & 3.908 & $0.331(-5)$ & 3.867 \\
\hline$v=0.10$ & 10 & $0.254(-2)$ & & $0.130(-1)$ & \\
\hline \multirow[t]{3}{*}{$\tau=0.0001$} & 20 & $0.137(-3)$ & 4.208 & $0.757(-3)$ & 4.110 \\
\hline & 40 & $0.958(-5)$ & 3.843 & $0.449(-4)$ & 4.075 \\
\hline & 80 & $0.635(-6)$ & 3.914 & $0.296(-5)$ & 3.921 \\
\hline
\end{tabular}

solving Burgers' equations is that shock of the solution may occur after some time, even if the initial functions are smooth. When the characteristic curves of Burgers' equation cross, a shock of the solution occurs. A robust and accurate numerical algorithm should be able to capture the shock and the numerical solution should exhibit the correct physical behavior. From Table 3, we observe that for small values of $v$, one must consider a large numbers of $N$ and $M$ to obtain proper solutions. Here our proposed scheme works well, and the orders of convergence of the proposed schemes are consistent with the theoretical expectations $O\left(h^{4}\right)$.

\section{Conclusion}

The proposed higher-order finite-difference schemes are easy for implementation and can be used for a numerical solution of two-dimensional coupled Burgers' equation with higher accuracy. The numerical results show that the variation in the values of the Reynolds number does not adversely affect the numerical solutions. Since all numerical results obtained by the above methods show a reasonably good agreement with the exact one for modest values of $v$, and also exhibit the expected convergence as the mesh size is decreased, the proposed methods can be considered to be competitive and worth recommendation.

\section{Acknowledgements}

The work was supported partially by the Foundation of Science and Technology of Mongolia, and the JINR theme 05-6-1119-2014/2016 "Methods, Algorithms and Software for Modeling Physical Systems, Mathematical Processing and Analysis of Experimental Data".

\section{References}

[1] Pandy, K., Lajja, V. and Amit, K.V. (2009) On a Finite-Difference Scheme for Burgers' Equation. Applied Mathematics and Computation, 215, 2208-2214. http://dx.doi.org/10.1016/j.amc.2009.08.018

[2] Srivastava, V.K., Tamsir, M., Bhardwaj, U. and Sanyasiraju, Y.V.S.S. (2011) Crank-Nicolson Scheme for Numerical Solution of Two-Dimensional Coupled Burgers' Equation. International Journal of Scientific and Engineering Research, 2, 2229-5518.

[3] Bahadir, A.R. (2003) A Fully Implicit Finite-Difference Scheme for Two-Dimensional Burgers' Equations. Applied Mathematics and Computation, 167, 131-137. http://dx.doi.org/10.1016/S0096-3003(02)00091-7

[4] Kweyu, M.C., Manyonge, W.A., Koross, A. and Ssemaganda, V. (2012) Numerical Solutions of the Burgers System in 
Two-Dimensions under Varied Initial and Boundary Conditions. Applied Mathematical Sciences, 6, 5603-5615.

[5] Tamsir, M. and Srivastava, V.K. (2011) A Semi-Implicit Finite-Difference Approach for Two Dimensional Coupled Burgers' Equations. International Journal of Scientific and Engineering Research, 2, 1-6.

[6] Srivastava, V.K., Awasthi, M.K. and Singh, S. (2013) An Implicit Logarithmic Finite-Difference Technique for Two Dimensional Coupled Viscous Burgers' Equation. AIP Advances, 3, 1-9.

[7] Zhao, G., Yu, X. and Zhang, R. (2011) The New Numerical Method for Solving the System of Two-Dimensional Burgers' Equations. Computers \& Mathematics with Applications, 62, 3279-3291. http://dx.doi.org/10.1016/j.camwa.2011.08.044

[8] Liao, W.Y. (2010) A Fourth Order Finite-Difference Method for Solving the System of Two-Dimensional Burgers' Equations. International Journal for Numerical Methods in Fluids, 64, 565-590. http://dx.doi.org/10.1002/fld.2163

[9] Cleophas, K., Benjamin, N. and John, W. (2014) Hybrid Crank-Nicolson-Du Fort and Frankel (CN-DF) Scheme for the Numerical Solution of the 2D Coupled Burgers' System. Applied Mathematical Sciences, 8, 2353-2361.

[10] Kalitkin, N.N. (1986) Numerical Methods. Nauka, Moscow. (In Russian)

[11] Zhanlav, T. (1978) Difference Schemes with Improved Accuracy for 1D Heat equation. Applied Mathematics, Irkutsk, 149-153. (In Russian)

[12] Fornberg, B. (1988) Generation of Finite Difference Formulas on Arbitrarily Spaced Grids. Mathematics of Computation, 51, 699-706. http://dx.doi.org/10.1090/S0025-5718-1988-0935077-0

[13] Ulziibayar, V., Zhanlav, T. and Chuluunbaatar, O. (2013) Higher-Order Accurate Numerical Solution of Burgers' Equation. International Journal of Mathematics Sciences, 33, 1374-1378.

[14] Zhanlav, T., Chuluunbaatar, O. and Ulziibayar, V. (2014) A Brief Description of Higher-Order Accurate Numerical Solution of Burgers' Equation. Series Mathematics, Information Sciences, Physics, 1, 86-91.

[15] Zhanlav, T., Chuluunbaatar, O. and Ulziibayar, V. (2015) Higher-Order Accurate Numerical Solution of Unsteady Burgers' Equation. Applied Mathematics and Computation, 250, 701-707. http://dx.doi.org/10.1016/j.amc.2014.11.013

[16] Samarskii, A.A. and Goolin, A.V. (1989) Numerical Methods. Nauka, Moscow. (In Russian)

[17] Mittal, R.C. and Jiwari, R. (2009) Differential Quadrature Method for Two-Dimensional Burgers' Equations. International Journal for Computational Methods in Engineering Science and Mechanics, 10, 450-459.

http://dx.doi.org/10.1080/15502280903111424 